\title{
Barrage laser photocoagulation for macula-sparing asymptomatic clinical rhegmatogenous retinal detachments
}

\begin{abstract}
Purpose To evaluate the efficacy of barrage laser photocoagulation in containing maculasparing asymptomatic clinical retinal detachments (RD).

Methods Consecutive patients presenting with asymptomatic clinical RD were prospectively treated with barrage photocoagulation in 2-3 confluent rows, using frequency-doubled Nd:YAG $(532 \mathrm{~nm})$ laser on an indirect-ophthalmoscopic delivery system. The patients were reviewed at $0.5,1.5,3$, and 6 months, and yearly thereafter. Best-corrected visual acuity (BCVA), and stability/ progression of rhegmatous retinal detachment beyond the barrage were noted at each visit.
\end{abstract}

Results Nineteen phakic eyes of 17 patients (nine female patients), aged 12-58 years (average: 26 years), underwent barrage laser treatment. Two women had bilateral RD. Most detachments were caused by atrophic holes, and involved at least a quadrant of retina. Seven $(37 \%)$ extended superiorly with breaks above the horizontal raphe. Three eyes had partial demarcation lines, and five had posterior vitreous detachment at presentation. The minimum follow-up was 6 months (mean: 21 months; range: 6-108 months). Pretreatment anatomical and functional status was maintained in $18(95 \%)$ eyes till the final visit. One superotemporal RD progressed across the laser barrier into macula 5 months after photocoagulation, and BCVA dropped to $6 / 18$. Scleral buckling was performed successfully, with visual recovery to $6 / 6$.

Conclusions Barrage photocoagulation may have a place in management of asymptomatic clinical detachments, as an effective and less morbid alternative to scleral buckling. Eye (2007) 21, 742-745; doi:10.1038/sj.eye.6702318; published online 17 March 2006

Keywords: barrage; photocoagulation; retinal detachment; demarcation; prophylaxis

Introduction

The decision to treat a disease is often based on the presence or absence of symptoms, one prototype case being rhegmatogenous retinal detachment (RRD). Literature is clear about the urgency of surgery for symptomatic RRD, but equivocal on the management of an asymptomatic detachment. ${ }^{1-4}$ The premise of symptom-based treatment is inherently unreliable owing to its subjective nature: a macula-sparing RRD may or may not cause symptoms, depending on its location, patient's vocational needs, and physical alertness / intelligence. For example, the article by Greven et $a l^{4}$ illustrates a large inferotemporal RRD encroaching into macula in an asymptomatic patient. Such a vision-threatening detachment cannot be conservatively managed merely because the patient is not aware of a superonasal scotoma.

We evaluated the protective effect of a limiting barrage photocoagulation in containing the progression of asymptomatic macula on clinical retinal detachments.

Patients and methods

This prospective, interventional case series enrolled outpatients presenting with clinical 
RRD (extending $>2$ disc-diameters (DD) posterior to the equator) ${ }^{5}$ without any symptoms (flashes, floaters,

shadows or vision loss). These patients either came for a routine evaluation or were referred by other ophthalmologists for vitreoretinal surgery - which was the primary treatment option offered to all the patients. Those who declined surgery were offered the option of barrage photocoagulation. The pros and cons of the two alternatives were clearly explained. We did not advise observation to any patient. The detachments bordering or involving macula, or those persisting/recurring after a vitreoretinal surgery were excluded from the study. Bullous superior detachments were similarly excluded. For all these categories, no alternative to vitreoretinal surgery was offered.

All underwent a comprehensive ophthalmic evaluation, including a dilated indirect ophthalmoscopy and biomicroscopy: the extent, topography and causative breaks of the retinal detachments were noted. The extent of posterior vitreous detachment (PVD) was assessed clinically, and confirmed by ultrasonography in doubtful cases. Total PVD was defined as the complete separation of posterior vitreous cortex from the retina and optic nerve head, visible as 'Weiss ring' on biomicroscopy, or as a low-reflectivity membrane with no retinal attachments and fluid, undulating after-movements on ultrasonography.
With informed consent of the patients and approval of the Institutional Review Board, barrage photocoagulation was performed in 2-3 rows of confluent burns with a $532 \mathrm{~nm}$ frequency-doubled Nd:YAG laser (Iridex Corp., Mountain View, CA, USA) using an indirectophthalmoscope mounted delivery system. Power (range: $250-400 \mathrm{~mW}$ ) and duration (0.1-0.2 s) were adjusted to obtain off-white burns; the detachments were barraged up to ora serrata (Figure 1). The patients were followed up at 15 days, 1.5, 3, and 6 months, and then yearly. Best-corrected visual acuity (BCVA) and stability of RRD were assessed at each visit.

\section{Results}

Nineteen eyes of 17 patients were included in the study; the average age was 26 years (range: $12-58$ years). There were eight male and nine female patients; two women had bilateral RRD. Out of 19 eyes, four were in the emmetropic range $( \pm 1 D)$, three had $1-3 \mathrm{D}$ of myopia, seven had 4-10 D, and five eyes had $>10 \mathrm{D}$ myopia. The causes of subnormal vision at presentation were myopic degeneration (five), amblyopia (three), and corneal opacities (one). All patients were phakic; none underwent cataract surgery during the follow-up. Five eyes had pre-existing total PVD at the time of presentation. The predominant causative lesion was

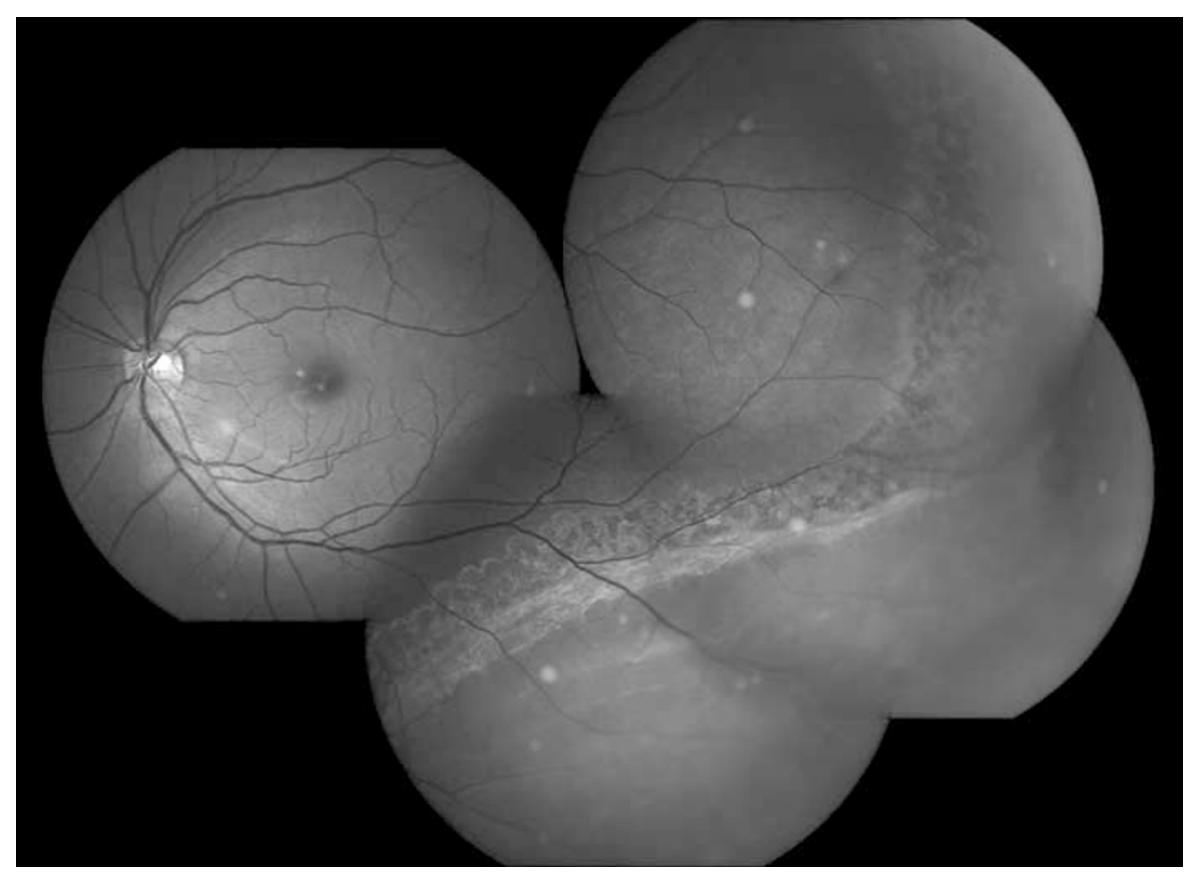

Figure 1 This 25-year-old lady was advised scleral buckling with vitrectomy for a two-quadrant macula-sparing retinal detachment that stopped just short of the arcades inferiorly. Her unaided vision was $6 / 6$ in the right and $6 / 12$ in the left eye. Barrage photocoagulation, as shown in the figure, stabilised the detachment over the follow-up of a year. The fellow eye was also found to have a clinical RRD, stabilised similarly by barrage photocoagulation (not illustrated). 
Table 1 Asymptomatic clinical detachments: clinical characteristics at presentation and post-barrage follow-up

\begin{tabular}{|c|c|c|c|c|c|c|c|c|c|c|}
\hline No. & Age (years) & Sex & $B C V A$ & $S E$ & $P V D$ & $R R D$ & Clock hours & Radial extent (DD) & Causative lesion & Follow-up (months) \\
\hline 1 & 25 & $\mathrm{~F}$ & $6 / 6$ & 3.0 & No & Inferior & 5 & 10 & Hole & 12 \\
\hline 2 & 25 & $\mathrm{~F}$ & $6 / 12$ & 3.0 & No & Inferior & 5 & 10 & Hole & 12 \\
\hline 3 & 19 & $\mathrm{~F}$ & $6 / 6$ & 7.0 & No & ST & 3 & 2 & Hole & 6 \\
\hline 4 & 24 & M & $6 / 6$ & 0.0 & No & Inferior & 3 & 2 & Hole & 9 \\
\hline 5 & 35 & $\mathrm{~F}$ & $6 / 12$ & 16.5 & No & Inferior & 4 & 10 & Hole & 6 \\
\hline 6 & 52 & $\mathrm{~F}$ & $6 / 12$ & 0.0 & Yes & Inferior & 5 & 10 & Lattice + hole & 12 \\
\hline 7 & 18 & M & $6 / 60$ & 3.75 & No & Inferior & 3 & 10 & Hole & 6 \\
\hline 8 & 18 & $\mathrm{~F}$ & $6 / 18$ & 6.5 & No & Inferior & 5 & 10 & Hole & 28 \\
\hline 9 & 18 & $\mathrm{~F}$ & $6 / 6$ & 6.5 & No & Inferior & 4 & 10 & Hole & 28 \\
\hline 10 & 58 & M & $6 / 18$ & 13.5 & Yes & Nasal & 3 & 6 & Hole & 6 \\
\hline 11 & 55 & $\mathrm{~F}$ & $6 / 12$ & 12.0 & Yes & Superior & 3 & 3 & Flap tear & 6 \\
\hline 12 & 35 & $\mathrm{M}$ & $6 / 6$ & 0.0 & Yes & Superior & 4 & 4 & Dialysis & 108 \\
\hline 13 & 15 & $\mathrm{~F}$ & $6 / 12$ & 12.0 & No & Temporal & 3 & 2 & Hole & 48 \\
\hline 14 & 18 & $\mathrm{~F}$ & $6 / 6$ & 5.25 & No & Superior & 4 & 2 & Lattice + hole & 6 \\
\hline 15 & 12 & M & $6 / 6$ & 0.0 & No & IT & 5 & 3 & Retinoschisis & 60 \\
\hline 16 & 14 & M & $6 / 6$ & 7.25 & No & Superior & 5 & 3 & Hole & 12 \\
\hline 17 & 23 & M & $6 / 6$ & 3.25 & Yes & Inferior & 5 & 2 & Dialysis & 14 \\
\hline 18 & 55 & $\mathrm{~F}$ & $6 / 6$ & 3.0 & Yes & IN & 3 & 3 & Lattice + hole & 12 \\
\hline 19 & 21 & M & $6 / 12$ & 12.5 & No & Temporal & 4 & 2 & Lattice + hole & 6 \\
\hline
\end{tabular}

BCVA: best-corrected visual acuity; SE: spherical equivalent (dioptres of myopia); PVD: total posterior vitreous detachment; RRD: retinal detachment; DD: disc diameters posterior to equator; ST: superotemporal; IT: inferotemporal; IN: inferonasal.

retinal hole, with or without lattice degeneration (16 eyes; $84 \%)$. One eye had outer- and inner-layer retinal holes in a peripheral retinoschisis. The other causative breaks were retinal dialyses (two) and flap tear (one). Seven eyes had primary retinal breaks above the horizontal raphe. The detachments were inferior (10), superior (five), temporal (three), or nasal (one). None of the detachments was bullous. The average extent of the RRD was 4 clockhours (range: 2-6 clock-hours) circumferentially, and 5.5 DD posterior to the equator (range: $2-10 \mathrm{DD}$ ). Three eyes had partial demarcation line at presentation. Two myopic patients (\#5 and 16) had RRD in the fellow eyes, the former (\#5) was spontaneously settled asymptomatic clinical RD with normal vision; the latter had undergone a successful scleral buckling (Table 1).

All eyes started and finished the study with the same visual acuity. The mean follow-up was 21 months (median: 12 months; range: 6-108 months). One eye (case 3) had a three-line fall in visual acuity owing to extension of a pre-existing superotemporal RD through the barrier photocoagulation into macula 5 months after treatment, without an associated PVD. It was successfully settled by scleral buckling, with recovery of vision to $6 / 6$.

\section{Discussion}

Over an average follow-up of 20.9 months, $95 \%$ of the asymptomatic clinical detachments in 17 patients (19 eyes) were successfully contained by barrage photocoagulation; the only one that progressed (into macula) was successfully repaired.
There is no consensus in literature on the management of asymptomatic clinical retinal detachments. Many authors forsake conservative approach once a subclinical RRD progresses to become clinical. ${ }^{4,6}$ Byer reported a variable amount of progress in 5/19 (26\%) subclinical detachments, although only two (10\%) became clinical, and required surgery. The rest did not require surgery in spite of progression probably because of their anterior location. ${ }^{6}$ Had the RRD already been close to arcades (as in a third of our cases), further progression would imply macular involvement. In the discussion of Byer's observations, Thompson mentioned extensive detachment, myopia, superior location, and absence of demarcation line as the risk factors of progression of RRD. ${ }^{6}$ These factors were present in most of our cases (vide infra).

Greven $e t a l^{4}$ also had RRD extending to arcades in a third of cases, and therefore preferred scleral buckling to observation. They reported an anatomical success rate of $93 \%$, and stabilised/improved vision in $85 \%$. These success rates are similar to our series. Surgical treatment, besides being expensive, has its associated morbidity, ranging from refractive errors and muscle imbalance, to retinal incarceration and ocular ischaemia. Therefore many asymptomatic patients hesitate to risk significant complications for only a prophylactic surgical measure.

Two large series on the natural history of asymptomatic clinical $\mathrm{RD}$ reported progression rates of $0-7 \%$ for such detachments. However, these studies reported a pre-existing PVD in 40-60\% eyes, demarcation lines in $74-100 \%$ of eyes, and superior breaks in $0-26 \%{ }^{2,3}$ 
The omnipresence of demarcation lines also biased these studies towards selecting detachments that were already longstanding at presentation, and therefore likely to remain so. In contrast, only $25 \%$ of our cases had PVD, $15 \%$ had demarcation lines at presentation, and $37 \%$ had superior breaks. Besides, $79 \%$ of our patients were myopic; and $37 \%$ of the detachments extended over two quadrants. A quarter of our patients were in fact referred to our hospital for vitreoretinal surgery. Therefore, we offered surgery as the first option to all our patients.

A critical given in observation of clinical RRD is an alert and compliant patient, who is expected to recognise the progression of RD, preferably before macular involvement, and report immediately. ${ }^{2}$ Such reliability of response is an unrealistic expectation in a vast, developing country like India, where tertiary care (eg scleral buckling) is expensive and sparse, and the patients are frequently ignorant of the blinding potential of vitreoretinal diseases. ${ }^{7}$ Thompson agreed that in view of unpredictable progression of RRD, some patients could lose sight permanently with surgical delay, and advised demarcating laser photocoagulation to reduce the urgency of follow-up. ${ }^{6}$

Yoon and Marmor reported that laser photocoagulation increased the strength of normal chorioretinal adhesion to 230-300\%. In contrast, spontaneously re-attached retina had only $75 \%$ of the normal adhesiveness. ${ }^{8}$ This strong adhesion develops over 2-4 weeks, which is therefore the recommended time interval for follow-up in these cases. ${ }^{1}$ When used as a prophylactic measure before silicone oil removal in post-vitrectomy eyes, laser encirclage also reduced the re-detachment rate by $50 \% .^{9}$ Vrabec and Baumal ${ }^{10}$ reported success with 'demarcation laser photocoagulation' of selected macula-sparing RRDs in a retrospective series. Only one out of 34 eyes (3\%) needed scleral buckling. However, they had a heterogeneous collection of phakic and pseudophakic, symptomatic and asymptomatic, and primary and recurrent detachments. They also did not report on the status of PVD before laser or during follow-up. Two earlier reports also reported success with similar laser photocoagulation in avoiding or at least delaying progression of RRD (14-30\%) and the need for vitrectomy in cytomegalovirus-related retinal detachments. ${ }^{11,12}$

Our series was uncontrolled; therefore, we cannot speculate on the comparative efficacy of barrage photocoagulation vis-à-vis vitreoretinal surgery or observation. As one of the detachments progressed after 5 months, we cannot rule out a similar progression in other eyes with further follow-up, especially after a PVD or cataract surgery. However, a possible indirect benefit of barrage photocoagulation is the early and accurate detection of RRD progression, as it begins to cross the laser barrier. This could be one of the reasons for excellent visual outcome in the case that required surgical intervention in our series. We therefore propose this modality as a viable intermediate treatment option, specifically for patients with macula-threatening clinical macular detachments who refuse or are physically unfit for vitreoretinal surgery. This technique may have a similar niche in the management of inferior/superior shallow RRD, as pneumoretinopexy has for superior RRD.

\section{References}

1 American Academy of Ophthalmology Retina Panel, Preferred Practice Patterns Committee. Retinal Detachment. American Academy of Ophthalmology (AAO): San Francisco, CA, 1990, pp 1-17.

2 Brod RD, Flynn Jr HW, Lightman DA. Asymptomatic rhegmatogenous retinal detachments. Arch Ophthalmol 1995; 113: 1030-1032.

3 Cohen SV. Natural history of asymptomatic rhegmatogenous retinal detachments. Am J Ophthalmol 2005; 139: 777-779.

4 Greven $\mathrm{CM}$, Wall AB, Slusher MM. Anatomic and visual results in asymptomatic clinical rhegmatogenous retinal detachment repaired by scleral buckling. Am J Ophthalmol 1999; 128: 618-620.

5 Davis MD. The natural history of retinal breaks without detachment. Trans Am Ophthalmol Soc 1973; 71: 343-372.

6 Byer NE. Subclinical retinal detachment resulting from asymptomatic retinal breaks: prognosis for progression and regression. Ophthalmology 2001; 108: 1499-1503, discussion 1503-1504.

7 Nirmalan PK, Katz J, Robin AL, Tielsch JM, Namperumalsamy P, Kim R et al. Prevalence of vitreoretinal disorders in a rural population of southern India: The Aravind Comprehensive Eye Study. Arch Ophthalmol 2004; 122: $581-586$

8 Yoon YH, Marmor MF. Rapid enhancement of retinal adhesion by laser photocoagulation. Ophthalmology 1988; 95 : 1385-1388.

9 Laidlaw DAH, Karia N, Bunce C, Aylward GW, Gregor ZJ. Is prophylactic $360^{\circ}$ laser retinopexy protective? Risk factors for retinal redetachment after removal of silicone oil. Ophthalmology 2002; 109: 153-158.

10 Vrabec TR, Baumal CR. Demarcation laser photocoagulation of selected macula-sparing rhegmatogenous retinal detachments. Ophthalmology 2000; 107: 1063-1067.

11 Vrabec TR. Laser photocoagulation repair of macula-sparing cytomegalovirus-related retinal detachment. Ophthalmology 1997; 104: 2062-2067.

12 Davis JL, Hummer J, Feuer WJ. Laser photocoagulation for retinal detachments and retinal tears in cytomegalovirus retinitis. Ophthalmology 1997; 104: 2053-2061. 